\title{
Unbalanced cluster sizes and rates of convergence in mixed-effects models for clustered data
}

\author{
W. Van der Elst, L. Hermans, G. Verbeke, M.G. Kenward, V. Nassiri \& G. \\ Molenberghs
}

To cite this article: W. Van der Elst, L. Hermans, G. Verbeke, M.G. Kenward, V. Nassiri \& G. Molenberghs (2015): Unbalanced cluster sizes and rates of convergence in mixed-effects models for clustered data, Journal of Statistical Computation and Simulation

To link to this article: http://dx.doi.org/10.1080/00949655.2015.1103738

\section{曲 Published online: 27 Oct 2015.}

Submit your article to this journal $\longleftarrow$

\section{Џ Article views: 23}

Q View related articles $\square$

View Crossmark data $\complement$ 


\title{
Unbalanced cluster sizes and rates of convergence in mixed-effects models for clustered data
}

\author{
W. Van der Elst ${ }^{a}$, L. Hermans $^{\mathrm{a}}$, G. Verbeke ${ }^{\mathrm{a}, \mathrm{b}}$, M.G. Kenward ${ }^{\mathrm{c}}$, V. Nassiri ${ }^{\mathrm{b}}$ and \\ G. Molenberghs $s^{a, b}$ \\ a-BioStat, Universiteit Hasselt, B-3590 Diepenbeek, Belgium; ${ }^{b}$ I-BioStat, KU Leuven, B-3000 Leuven, Belgium; \\ 'Department of Medical Statistics, London School of Hygiene and Tropical Medicine, London, UK
}

\begin{abstract}
Convergence problems often arise when complex linear mixed-effects models are fitted. Previous simulation studies (see, e.g. [Buyse M, Molenberghs G, Burzykowski T, Renard D, Geys H. The validation of surrogate endpoints in meta-analyses of randomized experiments. Biostatistics. 2000;1:49-67, Renard D, Geys H, Molenberghs G, Burzykowski T, Buyse M. Validation of surrogate end points in multiple randomized clinical trials with discrete outcomes. Biom J. 2002;44:921-935]) have shown that model convergence rates were higher (i) when the number of available clusters in the data increased, and (ii) when the size of the between-cluster variability increased (relative to the size of the residual variability). The aim of the present simulation study is to further extend these findings by examining the effect of an additional factor that is hypothesized to affect model convergence, i.e. imbalance in cluster size. The results showed that divergence rates were substantially higher for data sets with unbalanced cluster sizes - in particular when the model at hand had a complex hierarchical structure. Furthermore, the use of multiple imputation to restore 'balance' in unbalanced data sets reduces model convergence problems.
\end{abstract}

\section{ARTICLE HISTORY}

Received 20 January 2015

Accepted 28 September 2015

\section{KEYWORDS}

Simulation study; model convergence; mixed-effects model; multiple imputation; unbalanced data

\section{AMS SUBJECT CLASSIFICATION} 62J99; 62P10

\section{Introduction}

Fitting a linear mixed-effects model is typically done using Newton-Raphson or quasi-Newton-based procedures (for details, see [1]). Based on some starting values for the parameters at hand, these procedures iteratively update the parameter estimates until sufficient convergence is achieved. Unfortunately, non-converging iteration processes often occur when complex linear mixed-effects models are considered. This means that the iterative process does not converge at all, or that it converges to values that are close to or outside the boundary of the parameter space (i.e. variances that are close to zero or negative, which may lead to a non-positive-definite variance-covariance matrix of the random effects D). Such problems mainly occur in complex models with many covariance components.[2]

Motivating setting. As an example of a complex model with many covariance components where convergence is difficult to attain, consider the so-called surrogate endpoint evaluation setting. In a clinical trial, a surrogate endpoint is a replacement outcome for the true endpoint (i.e. the most credible indicator of treatment efficacy) that is useful when the latter endpoint is difficult to measure (e.g. infrequent, expensive, invasive, and/or distant in time).[3-5] Obviously, the surrogate endpoint $(S)$ can only replace the true endpoint $(T)$ when it has been formally evaluated. Nowadays, the metaanalytic framework is commonly used to statistically evaluate the appropriateness of a candidate 
S. [3,4] In this approach, it is assumed that information regarding $S$ and $T$ is available from multiple clinical trials (or from multiple other relevant units in which the patients are clustered, such as hospitals or countries; [6]). Based on these data, the following linear mixed-effects model is fitted:

$$
\begin{aligned}
S_{i j} & =\mu_{S}+m_{S i}+\left(\alpha+a_{i}\right) Z_{i j}+\varepsilon_{S i j}, \\
T_{i j} & =\mu_{T}+m_{T i}+\left(\beta+b_{i}\right) Z_{i j}+\varepsilon_{T i j},
\end{aligned}
$$

where $S_{i j}, T_{i j}$ refer to the (jointly normally distributed) surrogate and true endpoints for subject $j$ in cluster $i ; Z_{i j}$ is the binary treatment indicator for subject $j$ in cluster $i ; \mu_{S}, \mu_{T}$ are the fixed intercepts for $S$ and $T ; m_{S i}, m_{T i}$ are the corresponding random intercepts; $\alpha, \beta$ are the fixed treatment effects for $S$ and $T ; a_{i}, b_{i}$ are the corresponding random effects, and $\varepsilon_{S i j}, \varepsilon_{T i j}$ are the error terms for $S$ and $T$, respectively. It is assumed that $\left(m_{S i}, m_{T i}, a_{i}, b_{i}\right) \sim N(\mathbf{0}, \mathbf{D})$ and $\left(\varepsilon_{S i j}, \varepsilon_{T i j}\right) \sim N(\mathbf{0}, \boldsymbol{\Sigma})$, where $\mathbf{D}$ and $\boldsymbol{\Sigma}$ are unstructured variance-covariance matrices.

The appropriateness of a candidate surrogate is quantified by two metrics. First, the coefficient of trial-level surrogacy $\left(R_{\text {trial }}^{2}\right)$, which essentially quantifies the strength of the association between the random treatment effects on $S$ and $T$ based on the variance-covariance matrix of the random effects $\mathbf{D}$, i.e. $R_{\text {trial }}^{2}=d_{a b}^{2} / d_{a a} d_{b b}$. Second, the coefficient of individual-level surrogacy $\left(R_{\text {indiv }}^{2}\right)$, which quantifies the treatment- and trial-corrected strength of association between $S$ and $T$ based on the variance-covariance matrix of the residuals $\Sigma$, i.e. $R_{\text {indiv }}^{2}=\sigma_{S T}^{2} / \sigma_{S S} \sigma_{T T}$.

Earlier simulation studies. In a surrogate evaluation context, the $(S, T)$ endpoints (level 1$)$ are nested within patients (level 2), and the patients are nested within clinical trials (or other relevant clustering units; level 3). Given the relatively complex hierarchical structure of the data, it is hardly surprising that convergence problems are frequently encountered in a surrogate evaluation context.

To gain more insight into the factors that affect model convergence, Buyse et al. [3] and Renard et al. [7] conducted a number of simulation studies. Their conclusion was that model convergence rates were higher when the number of available clusters increased and when the size of the between-cluster variability $\mathbf{D}$ increased relative to the residual variability $\Sigma$ (in particular the $d_{a a}, d_{b b}$ components relative to the $\sigma_{S S}, \sigma_{T T}$ components). Other factors, such as the number of patients per cluster, the normality assumption (for $S$ and $T$ ), and the strength of the correlation between the random treatment effects had no substantial impact on model convergence.

Aim of the present study. The aim of the present study is to further extend the results of the earlier simulation studies $[3,7]$ by evaluating the effect of an additional factor that may affect model convergence, i.e. imbalance in cluster size. Indeed, in the earlier simulation studies, balanced data sets were considered, i.e. all clusters had exactly the same number of observations. However, in real-life data sets, it is nearly always the case that the cluster sizes are unbalanced. In fact, even when a balanced design was initially planned, the actually collected data will often be unbalanced due to, for example, missingness.

To understand why balance in cluster size may be a relevant factor to consider, recall that the key computational difficulty in fitting mixed-effects models is in the estimation of the covariance parameters. [2] Iterative numerical optimization of the log-likelihood functions using (RE)ML estimation is conducted, subject to constraints that are imposed on the model parameters to ensure positive definiteness of the $\mathbf{D}$ and $\mathbf{V}_{i}=\mathbf{Z}_{i} \mathbf{D} \mathbf{Z}_{i}^{\prime}+\boldsymbol{\Sigma}_{i}$ matrices (where $\mathbf{Z}_{i}$ are matrices of known covariates associated with the random effects). Notice that positive definiteness of both $\mathbf{D}$ and $\mathbf{V}_{i}$ is not needed when one is merely interested in the marginal model. In the latter case, the only requirement for valid inference based on the marginal model is that the overall $\mathbf{V}$ matrix is positive definite (PD)(Section 5). To maximize complicated likelihoods or to find good starting values that can subsequently be used in the Newton-Raphson algorithm, the expectation-maximization (EM) algorithm is often used.[8] When unbalanced data are considered, the E-step involves, at least conceptually, the creation of a 'balanced' data set (or a 'complete' data set in a missing data context [9]) based on a hypothetical scenario where it is assumed that data have been obtained from a balanced design (or from a study in which there were no missing values in a missing data context $[2,10]$ ). Based on the 
'balanced' data, an objective function is constructed and maximized in the M-step, and the parameter estimates are subsequently iteratively updated. In essence, the underlying assumption behind the EM algorithm is that the optimization of the balanced (complete) data log-likelihood function is easier than the optimization of the unbalanced (observed) data log-likelihood.[10] In the same spirit, it can be expected that model convergence issues will occur more frequently when the actually observed data are unbalanced in cluster size, compared to the setting where the actually observed data are balanced. The first aim of the present study is to examine this hypothesis.

The second aim of the present study is to examine whether the convergence rates of unbalanced data could be increased by using multiple imputation (MI) prior to fitting the mixed-effects model. Based on the reasoning in the previous paragraph, it was expected that the use of MI (to make an unbalanced data set 'balanced') would lead to higher convergence rates.

Organization of the paper. The remainder of this paper is organized as follows. In Section 2, the impact of imbalance in cluster size on the convergence rates of mixed-effects models is evaluated. In Section 3, the impact of using MI to introduce balance in unbalanced data sets on model convergence rates is examined and the statistical properties of the estimators are evaluated. In Section 4, a case study (the age-related macular degeneration trial) is analysed. Finally, Section 5 summarizes the results and discusses some of the limitations of the present paper.

\section{Unbalanced data and model convergence}

Three mixed-effects models with an increasing level of complexity were considered: (i) a randomintercept model, (ii) a reduced surrogate evaluation model (i.e. a simplification of Model (1) where the fixed- and random-treatment effects are discarded), and (iii) a surrogate evaluation model (Model (1)). The idea is to gradually build-up the (hierarchical) complexity of the model, so that it can be examined at which level of complexity the impact of an imbalance in cluster size on model convergence becomes apparent.

\subsection{Outcomes of interest}

The key outcome of interest in the simulations was model convergence. Three model convergence categories were distinguished: (i) proper convergence, i.e. the model converged and the variance-covariance matrix of the random effects $(\mathbf{D})$ and the final Hessian $(\mathbf{H})$, used to compute the standard errors of the covariance parameters, were PD ; (ii) the model converged but $\mathbf{D}$ or $\mathbf{H}$ was not PD; and finally, (iii) divergence. In addition, the number of required iterations to achieve convergence was recorded and analysed.

\subsection{Simulation design}

\subsubsection{Random-intercept model}

Consider the following random-intercept model:

$$
S_{i j}=\mu_{S}+m_{S i}+\varepsilon_{S i j}
$$

where $S_{i j}$ is a (normally distributed) endpoint for patient $j$ in cluster $i, \mu_{S}$ is the fixed intercept, $m_{S i}$ is the corresponding random intercept, and $\varepsilon_{S i j}$ is the error term. It is assumed that $m_{S i} \sim N(0, d)$ and $\varepsilon_{S i j} \sim N\left(0, \sigma_{S S}\right)$.

In all simulations, $\mu_{S}=450, \sigma_{S S}=300$, and the mean sample size per cluster $M\left(n_{i}\right)=20$. Three conditions were varied. First, the number of clusters $i=1,2, \ldots, N$, with $N=\{5,10,20,50\}$. Second, the level of imbalance in cluster size $\left(n_{i}\right)$. In the balanced scenario, all cluster sizes were equal, i.e. $n_{i}=n=20$. In the two unbalanced scenarios, $\tilde{n}_{i}$ was determined based on a draw from a normal distribution and rounded to the nearest integer (i.e. $n_{i}=\operatorname{round}\left(\tilde{n}_{i}\right)$ ). In 
the low imbalance scenario, $\tilde{n}_{i} \sim N\left(20,2.5^{2}\right)$. In the high imbalance scenario, $\tilde{n}_{i} \sim N\left(20,5^{2}\right)$. Third, the between-cluster variability $(d=\gamma(1000))$, which is either large $(\gamma=1)$ or small $(\gamma=0.1)$ relative to the residual variability $\left(\sigma_{S S}=300\right)$. There were thus a total of 24 possible scenarios, for each of which 1000 data sets were generated. These data sets were subsequently analysed by fitting Model (2) using the mixed procedure in statistical analysis system (SAS), and model convergence and the required number of iterations were recorded (Section 2.1).

\subsubsection{Reduced surrogate evaluation model}

Consider the following linear mixed-effects model:

$$
\begin{aligned}
& S_{i j}=\mu_{S}+m_{S i}+\varepsilon_{S i j}, \\
& T_{i j}=\mu_{T}+m_{T i}+\varepsilon_{T i j},
\end{aligned}
$$

where $S_{i j}$ and $T_{i j}$ are (normally distributed) endpoints for patient $j$ in cluster $i$ (e.g. a surrogate and true endpoint); $\mu_{S}, \mu_{T}$ are the fixed intercepts for $S$ and $T ; m_{S i}, m_{T i}$ are the corresponding random intercepts; and $\varepsilon_{S i j}, \varepsilon_{T i j}$ are the error terms. It is assumed that $\left(m_{S i}, m_{T i}\right) \sim N(\mathbf{0}, \mathbf{D})$ and $\left(\varepsilon_{S i j}, \varepsilon_{T i j}\right) \sim N(\mathbf{0}, \boldsymbol{\Sigma})$, where $\mathbf{D}$ and $\boldsymbol{\Sigma}$ are unstructured 2 by 2 variance-covariance matrices. As can be seen, Model (3) is a simplification of Model (1), where the fixed- and random- treatment effects are omitted.

Using Model (3), data were simulated. In all simulations, $\mu_{S}=450, \mu_{T}=500$, and

$$
\boldsymbol{\Sigma}=\left(\begin{array}{cc}
300 & 212.132 \\
212.132 & 300
\end{array}\right)
$$

yielding $\operatorname{corr}\left(\varepsilon_{S i j}, \varepsilon_{T i j}\right)^{2}=0.5$. Three conditions were varied in the simulations. First, the number of clusters $N=\{5,10,20,50\}$. Second, the level of imbalance in $n_{i}$ (see Section 2.2.1). Third, the between-cluster variability $(\mathbf{D})$, which is either large $(\gamma=1)$ or small $(\gamma=0.1)$ relative to the residual variability $(\boldsymbol{\Sigma})$ :

$$
\mathbf{D}=\gamma\left(\begin{array}{cc}
1000 & 400 \\
400 & 1000
\end{array}\right) .
$$

For each of the 24 settings, a total of 1000 data sets were generated, and model convergence and the required number of iterations were recorded (Section 2.1).

\subsubsection{Surrogate evaluation model}

Using the linear mixed-effects Model (1) detailed above, data were simulated. Notice that $Z_{i j}$ was coded as $-1=$ control treatment and $1=$ experimental treatment (rather than as $0=$ control treatment and $1=$ experimental treatment), and thus the fixed treatment effects on $S$ and $T$ are $2 \alpha$ and $2 \beta$, respectively. This was done because a $0 / 1$ coding, for a positive-definite $\mathbf{D}$ matrix, forces the variability in the experimental arm to be greater than or equal to the variability in the control arm. A $-1 / 1$ coding on the other hand ensures that the same components of variability operate in both treatment arms. [4]

In all simulations, $\mu_{S}=450, \mu_{T}=500, \alpha=300, \beta=500$, and

$$
\boldsymbol{\Sigma}=\left(\begin{array}{cc}
300 & 212.132 \\
212.132 & 300
\end{array}\right)
$$

yielding $R_{\text {indiv }}^{2}=\operatorname{corr}\left(\varepsilon_{S i j}, \varepsilon_{T i j}\right)^{2}=0.5$. Again, three conditions were varied in the simulations. First, the number of clusters $N=\{5,10,20,50\}$. Second, the level of imbalance in $n_{i}$ (see Section 2.2.1). 
Third, the between-cluster variability $(\mathbf{D})$, which is either large $(\gamma=1)$ or small $(\gamma=0.1)$ relative to the residual variability $(\Sigma)$ :

$$
\mathbf{D}=\gamma\left(\begin{array}{cccc}
1000 & 400 & 0 & 0 \\
400 & 1000 & 0 & 0 \\
0 & 0 & 1000 & 707.107 \\
0 & 0 & 707.107 & 1000
\end{array}\right)
$$

yielding $R_{\text {trial }}^{2}=\operatorname{corr}\left(a_{i}, b_{i}\right)^{2}=0.5$. Furthermore, in the balanced scenario, treatment $(Z)$ is also balanced within a cluster. In the unbalanced scenarios, treatment allocation is determined based on a binomial distribution with success probability 0.50 . A total of 1000 data sets were generated for each of the 24 settings. The generated data were subsequently analysed by fitting Model (1) in SAS. Two different parametrizations for the $\mathbf{D}$ matrix were considered. First, a completely general (unstructured; UN) D matrix that is parameterized directly in terms of variances and covariances. Second, a nondiagonal factor analytic structure with 4 factors (FA0(4)). The latter structure specifies a Cholesky root parametrization for the $4 \times 4$ unstructured blocks in $\mathrm{D}$. This leads to a substantial simplification of the optimization problem, i.e. the problem now changes from a constrained one to an unconstrained one. The FA0(4) structure has $(q / 2)(2 t-q+1)$ covariance parameters, where $q$ refers to the number of factors and $t$ is the dimension of the matrix. In the present setting, the FA0(4) structure thus has a total of 10 parameters. These parameters are used to compute the components in $\mathbf{D}$, i.e. the $(i, j)$ th element of $\mathbf{D}$ is computed as $\Sigma_{k=1}^{\min (i, j, k)} \lambda_{i k} \lambda_{j k}$. The Cholesky root parametrization ensures that $\mathbf{D}$ (and $\mathbf{V}_{i}$ ) is positive definite during the entire estimation process.[10] Model convergence and the required number of iterations were recorded (Section 2.1).

\subsection{Results}

As shown in Table 1, the rates of proper convergence exceeded 0.960 and 0.841 in the various scenarios for the random-intercept and reduced surrogate models, respectively. Overall convergence (i.e. proper convergence or convergence but non-PD D or $\mathbf{H}$ matrix) was $100 \%$ for the random-intercept model and $\geq 97.8 \%$ for the reduced surrogate model. The rates of proper and overall convergence were similar in all scenarios, irrespective of the level of imbalance in cluster size $\left(n_{i}\right)$. However, a larger level of imbalance in cluster size was associated with a higher mean number of iterations that were required to achieve proper convergence for both the random-intercept and the reduced surrogate models (see Table 2). This suggests that the optimization of the log-likelihood function is more difficult in the unbalanced scenarios, even when relatively simple (in terms of their hierarchical structure) mixedeffects models are considered.

When an unstructured (UN) D matrix was used in the surrogate evaluation models, overall convergence exceeded $99.7 \%$ when cluster sizes were balanced (Table 1). The overall convergence rates were, however, substantially lower in the unbalanced scenarios, in particular when $N$ and $\gamma$ were small. For example, when $N=5$ and $\gamma=0.1$, the model divergence rates were as high as $65.5 \%$ and $77.3 \%$ in the small and large imbalance scenarios, respectively (compared to only $0.3 \%$ in the balanced scenario). At the same time, the impact of level of imbalance on proper convergence was small, i.e. proper convergence rates were quite similar in all scenarios irrespective of the level of imbalance in the data. When a non-diagonal factor analytic structure with 4 factors (FA0(4)) was used for the D matrix in the surrogate evaluation models, the rates of proper convergence exceeded $71.0 \%$ in all scenarios (Table 1) and were thus substantially higher compared to those that were observed in the UN scenario. Furthermore, divergence rates were substantially lower in the unbalanced FA0(4) scenarios compared to those in the unbalanced UN scenarios. As was also observed for the random-intercept and the reduced surrogate models, a higher level of imbalance in cluster size was associated with a larger mean number of required iterations to achieve proper convergence for both the UN and FA0(4) surrogate evaluation models (Table 2). A noteworthy observation is that proper convergence was 


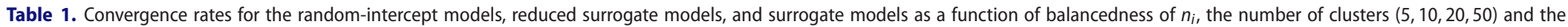
between-cluster variability.

\begin{tabular}{|c|c|c|c|c|c|c|c|c|c|c|c|c|c|c|}
\hline \multirow[b]{2}{*}{ Model } & \multirow[b]{2}{*}{$\begin{array}{l}\text { Convergence } \\
\text { category }\end{array}$} & \multirow{2}{*}{$\begin{array}{l}\text { Between- } \\
\text { cluster } \\
\text { variability } \gamma\end{array}$} & \multicolumn{4}{|c|}{$\begin{array}{l}\text { Balanced (equal } n_{i} \text { ) } \\
\text { Number of clusters }\end{array}$} & \multicolumn{4}{|c|}{$\begin{array}{c}\text { Small imbalance } \\
\tilde{n}_{i} \sim N\left(20,2.5^{2}\right) \\
\text { Number of clusters }\end{array}$} & \multicolumn{4}{|c|}{$\begin{array}{c}\text { Large imbalance } \\
\tilde{n}_{i} \sim N\left(20,5^{2}\right) \\
\text { Number of clusters }\end{array}$} \\
\hline & & & 5 & 10 & 20 & 50 & 5 & 10 & 20 & 50 & 5 & 10 & 20 & 50 \\
\hline \multirow[t]{6}{*}{ Random intercept } & Proper convergence & Small (0.1) & 0.974 & 0.999 & 1 & 1 & 0.969 & 0.999 & 1 & 1 & 0.960 & 0.998 & 1 & 1 \\
\hline & & Large (1) & 0.999 & 1 & 1 & 1 & 0.997 & 1 & 1 & 1 & 0.989 & 1 & 1 & 1 \\
\hline & $\begin{array}{l}\text { Convergence but } \\
\text { non-PD D/H matrix }\end{array}$ & Small (0.1) & 0.026 & 0.001 & 0 & 0 & 0.031 & 0.001 & 0 & 0 & 0.040 & 0.002 & 0 & 0 \\
\hline & & Large (1) & 0.001 & 0 & 0 & 0 & 0.003 & 0 & 0 & 0 & 0.011 & 0 & 0 & 0 \\
\hline & Divergence & Small (0.1) & 0 & 0 & 0 & 0 & 0 & 0 & 0 & 0 & 0 & 0 & 0 & 0 \\
\hline & & Large (1) & 0 & 0 & 0 & 0 & 0 & 0 & 0 & 0 & 0 & 0 & 0 & 0 \\
\hline \multirow{6}{*}{$\begin{array}{l}\text { Reduced surrogate } \\
\text { model }\end{array}$} & Proper convergence & Small (0.1) & 0.853 & 0.991 & 1 & 1 & 0.853 & 0.990 & 1 & 1 & 0.841 & 0.987 & 1 & 1 \\
\hline & & Large (1) & 0.990 & 1 & 1 & 1 & 0.992 & 1 & 1 & 1 & 0.995 & 1 & 1 & 1 \\
\hline & $\begin{array}{l}\text { Convergence but } \\
\text { non-PD D/H matrix }\end{array}$ & Small (0.1) & 0.147 & 0.009 & 0 & 0 & 0.141 & 0.010 & 0 & 0 & 0.137 & 0.013 & 0 & 0 \\
\hline & & Large (1) & 0.010 & 0 & 0 & 0 & 0.007 & 0 & 0 & 0 & 0.004 & 0 & 0 & 0 \\
\hline & Divergence & Small (0.1) & 0 & 0 & 0 & 0 & 0.006 & 0 & 0 & 0 & 0.022 & 0 & 0 & 0 \\
\hline & & Large (1) & 0 & 0 & 0 & 0 & 0.001 & 0 & 0 & 0 & 0.001 & 0 & 0 & 0 \\
\hline \multirow[t]{6}{*}{ Surrogate model, UN } & Proper convergence & Small (0.1) & 0.112 & 0.826 & 0.999 & 1 & 0.090 & 0.816 & 1 & 1 & 0.091 & 0.800 & 0.999 & 1 \\
\hline & & Large (1) & 0.555 & 0.998 & 1 & 1 & 0.566 & 0.999 & 1 & 1 & 0.540 & 0.996 & 1 & 1 \\
\hline & $\begin{array}{l}\text { Convergence but } \\
\text { non-PD D/H matrix }\end{array}$ & Small (0.1) & 0.885 & 0.174 & 0.001 & 0 & 0.255 & 0.171 & 0 & 0 & 0.136 & 0.155 & 0.001 & 0 \\
\hline & & Large (1) & 0.444 & 0.002 & 0 & 0 & 0.163 & 0.001 & 0 & 0 & 0.119 & 0.001 & 0 & 0 \\
\hline & Divergence & Small (0.1) & 0.003 & 0 & 0 & 0 & 0.655 & 0.013 & 0 & 0 & 0.773 & 0.045 & 0 & 0 \\
\hline & & Large (1) & 0.001 & 0 & 0 & 0 & 0.271 & 0 & 0 & 0 & 0.341 & 0.003 & 0 & 0 \\
\hline \multirow{6}{*}{$\begin{array}{l}\text { Surrogate model, } \\
\text { FAO(4) }\end{array}$} & Proper convergence & Small (0.1) & 0.745 & 0.984 & 1 & 1 & 0.717 & 0.976 & 1 & 1 & 0.710 & 0.976 & 0.999 & 1 \\
\hline & & Large (1) & 0.931 & 1 & 1 & 1 & 0.931 & 0.994 & 0.997 & 0.998 & 0.935 & 0.992 & 0.998 & 0.999 \\
\hline & $\begin{array}{l}\text { Convergence but } \\
\text { non-PD D/H matrix }\end{array}$ & Small (0.1) & 0.068 & 0.007 & 0 & 0 & 0.054 & 0.016 & 0 & 0 & 0.044 & 0.015 & 0 & 0 \\
\hline & & Large (1) & 0.030 & 0 & 0 & 0 & 0.027 & 0.001 & 0.002 & 0 & 0.018 & 0.004 & 0.001 & 0.001 \\
\hline & Divergence & Small (0.1) & 0.187 & 0.009 & 0 & 0 & 0.229 & 0.008 & 0 & 0 & 0.246 & 0.009 & 0.001 & 0 \\
\hline & & Large (1) & 0.039 & 0 & 0 & 0 & 0.042 & 0.005 & 0.001 & 0.002 & 0.047 & 0.004 & 0.001 & 0 \\
\hline
\end{tabular}

Note: $\mathrm{UN}=$ unstructured; $\mathrm{FAO}(4)=$ factor analytic. 


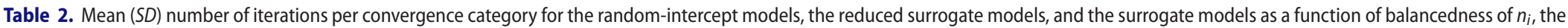
number of clusters $(5,10,20,50)$ and the between-cluster variability.

\begin{tabular}{|c|c|c|c|c|c|c|c|c|c|c|c|c|c|c|}
\hline \multirow[b]{2}{*}{ Model } & \multirow[b]{2}{*}{$\begin{array}{c}\text { Convergence } \\
\text { category }\end{array}$} & \multirow{2}{*}{$\begin{array}{c}\text { Between- } \\
\text { cluster } \\
\text { variability } \gamma\end{array}$} & \multicolumn{4}{|c|}{ Balanced (equal $n_{i}$ ) } & \multicolumn{4}{|c|}{$\begin{array}{l}\text { Small imbalance } \\
\tilde{n}_{i} \sim N\left(20,2.5^{2}\right) \\
\text { Number of clusters }\end{array}$} & \multicolumn{4}{|c|}{$\begin{array}{c}\text { Large imbalance } \\
\tilde{n}_{i} \sim N\left(20,5^{2}\right) \\
\text { Number of clusters }\end{array}$} \\
\hline & & & 5 & 10 & 20 & 50 & 5 & 10 & 20 & 50 & 5 & 10 & 20 & 50 \\
\hline Random & Proper convergence & Small (0.1) & $1(0)$ & $1(0)$ & $1(0)$ & $1(0)$ & $2.18(0.85)$ & $2.22(0.80)$ & $2.09(0.73)$ & $1.88(0.63)$ & $2.70(1.06)$ & $2.71(0.95)$ & $2.52(0.87)$ & $2.32(0.78)$ \\
\hline \multirow[t]{5}{*}{ intercept } & & Large (1) & $1(0)$ & $1(0)$ & $1(0)$ & $1(0)$ & $2.91(1.31)$ & $2.95(1.14)$ & $2.82(1.02)$ & $2.56(0.84)$ & $3.70(1.89)$ & $3.85(1.85)$ & $3.64(1.68)$ & $3.13(1.04)$ \\
\hline & $\begin{array}{l}\text { Convergence but } \\
\text { non-PD D/H matrix }\end{array}$ & Small (0.1) & $1(0)$ & $1(-)$ & - & - & $1(0)$ & $1(-)$ & - & - & $1(0)$ & $1(0)$ & - & - \\
\hline & & Large (1) & $1(-)$ & - & - & - & $1(0)$ & - & - & - & $1(0)$ & - & - & - \\
\hline & Divergence & Small (0.1) & - & - & - & - & - & - & - & - & - & - & - & - \\
\hline & & Large (1) & - & - & - & - & - & - & - & - & - & - & - & - \\
\hline $\begin{array}{l}\text { Reduced } \\
\quad \text { surrogate }\end{array}$ & Proper convergence & Small (0.1) & $1(0)$ & $1(0)$ & $1(0)$ & $1(0)$ & $2.67(0.78)$ & $2.59(0.70)$ & $2.35(0.58)$ & $2.08(0.44)$ & $3.51(1.02)$ & $3.37(0.89)$ & $3.06(0.77)$ & $2.66(0.65)$ \\
\hline \multirow[t]{5}{*}{ model } & & Large (1) & $1(0)$ & $1(0)$ & $1(0)$ & $1(0)$ & $4.06(1.78)$ & $4.12(1.72)$ & $3.84(1.35)$ & $3.32(0.79)$ & $5.36(2.30)$ & $5.42(2.32)$ & $5.11(2.36)$ & $4.28(1.51)$ \\
\hline & $\begin{array}{l}\text { Convergence but } \\
\text { non-PD D/H matrix }\end{array}$ & Small (0.1) & $4.28(1.61)$ & $3.22(0.67)$ & - & - & $4.38(1.47)$ & $4.10(0.88)$ & - & - & $4.46(1.44)$ & $4.15(1.46)$ & - & - \\
\hline & & Large (1) & $4.20(1.40)$ & - & - & - & $4.14(1.35)$ & - & - & - & $5.50(2.53)$ & - & - & - \\
\hline & Divergence & Small (0.1) & - & - & - & - & $36.17(6.31)$ & - & - & - & $50.46(58.29)$ & - & - & - \\
\hline & & Large (1) & - & - & - & - & $44.00(-)$ & - & - & - & $36.00(-)$ & - & - & - \\
\hline \multirow{6}{*}{$\begin{array}{l}\text { Surrogate model, } \\
\text { UN }\end{array}$} & Proper convergence & Small (0.1) & $1(0)$ & $1(0)$ & $1(0)$ & $1(0)$ & $4.99(1.14)$ & $4.30(0.85)$ & $3.70(0.73)$ & $3.11(0.58)$ & $5.34(1.12)$ & $4.72(0.86)$ & $4.15(0.70)$ & $3.52(0.67)$ \\
\hline & & Large (1) & $1(0)$ & $1(0)$ & $1(0)$ & $1(0)$ & $7.68(2.60)$ & $6.73(2.49)$ & $5.84(2.43)$ & $4.69(1.75)$ & $8.21(2.40)$ & $7.35(2.67)$ & $6.67(2.63)$ & $5.54(2.32)$ \\
\hline & $\begin{array}{l}\text { Convergence but } \\
\text { non-PD D/H matrix }\end{array}$ & Small (0.1) & $9.16(9.06)$ & $3.97(1.37)$ & $4(-)$ & - & $6.85(2.13)$ & $5.34(1.34)$ & - & - & $6.73(1.83)$ & $5.56(1.32)$ & $5(-)$ & - \\
\hline & & Large (1) & $6.35(3.50)$ & $3.50(2.12)$ & - & - & $9.90(2.71)$ & $8.00(-)$ & - & - & $9.57(2.61)$ & $8.00(-)$ & - & - \\
\hline & Divergence & Small (0.1) & $309.33(306.91)$ & - & - & - & $50.10(16.51)$ & $49.00(12.17)$ & - & - & $47.66(15.01)$ & $48.81(16.05)$ & - & - \\
\hline & & Large (1) & $205.00(-)$ & - & - & - & $50.79(19.71)$ & - & - & - & $49.40(15.39)$ & $44.00(2.00)$ & - & - \\
\hline \multirow{6}{*}{$\begin{array}{l}\text { Surrogate model, } \\
\text { FA0(4) }\end{array}$} & Proper convergence & Small $(0.1)$ & $4.90(3.90)$ & $1.66(1.87)$ & $1(0.09)$ & $1(0)$ & $6.00(2.99)$ & $4.37(3.16)$ & $2.87(0.62)$ & $2.36(0.49)$ & $6.45(3.31)$ & $4.94(3.38)$ & $3.29(1.20)$ & $2.77(0.52)$ \\
\hline & & Large (1) & $2.24(1.19)$ & $1.01(0.16)$ & $1(0)$ & $1(0)$ & $9.54(7.69)$ & $6.36(3.42)$ & $5.67(3.33)$ & $4.65(2.27)$ & $10.87(8.57)$ & $6.98(4.54)$ & $6.27(3.08)$ & $5.40(2.61)$ \\
\hline & $\begin{array}{l}\text { Convergence but } \\
\text { non-PD D/H matrix }\end{array}$ & Small (0.1) & $6.12(1.94)$ & $5.71(2.21)$ & - & - & $6.91(2.05)$ & $5.69(1.45)$ & - & - & $6.55(1.30)$ & $5.80(1.93)$ & - & - \\
\hline & & Large (1) & $5.13(1.68)$ & - & - & - & $11.85(7.48)$ & $20(-)$ & $23.50(0.71)$ & - & $13.39(14.14)$ & $24(16.67)$ & $23(-)$ & $38(-)$ \\
\hline & Divergence & Small (0.1) & $21.96(13.84)$ & $30.78(12.13)$ & - & - & $19.41(11.75)$ & $18.25(9.77)$ & - & - & $20.10(12.88)$ & $32.33(18.32)$ & $49(0)$ & - \\
\hline & & Large (1) & $31.67(11.43)$ & - & - & - & $29.57(13.46)$ & $27(11.98)$ & $11(-)$ & 17(5.66) & $27.87(11.82)$ & $29.50(21.61)$ & $9(-)$ & - \\
\hline
\end{tabular}

Note: $\mathrm{UN}=$ unstructured; $\mathrm{FAO}(4)=$ factor analytic. 
always achieved after 1 iteration when the cluster size was balanced for the random-intercept, reduced surrogate, and UN surrogate evaluation models (Table 2). The reverse also holds approximately, i.e. when a model converged after 1 iteration, in more than $99.9 \%$ of the cases there was proper convergence. In contrast to what was the case for the UN surrogate evaluation models, proper convergence was not always achieved after 1 iteration for the FA0(4) surrogate evaluation models. Nonetheless, the number of required iterations to achieve proper convergence was also substantially lower in the balanced FA0(4) surrogate evaluation models compared to what was the case in the unbalanced FA0(4) surrogate evaluation models.

\section{Multiple imputation}

\subsection{Simulation design}

The same unbalanced data sets that were generated in the surrogate evaluation model scenario described above (Section 2.2.3) were considered here. In these unbalanced data sets, MI was used to introduce balance in terms of cluster size and treatment allocation (Z). As an example of what is meant by this, consider the hypothetical data set with five clusters shown in Table 3. As can be seen, the maximum number of patients for each of the cluster by treatment $(Z)$ groups is 18 . Thus for all cluster $\times$ treatment groups having less than 18 observations, MI is used to restore balance.

Table 3. Hypothetical dataset. Number of observations per cluster as a function of treatment (Z), before and after imputation.

\begin{tabular}{lccccc}
\hline & \multicolumn{2}{c}{ Before imputation } & & \multicolumn{2}{c}{ After imputation } \\
\cline { 2 - 3 } \cline { 5 - 6 } Cluster & $Z=-1$ & $Z=1$ & & $Z=-1$ & $Z=1$ \\
\hline 1 & 5 & 11 & 18 & 18 \\
2 & 13 & 8 & 18 & 18 \\
3 & 10 & 18 & 18 & 18 \\
4 & 9 & 5 & 18 & 18 \\
5 & 9 & 11 & 18 & \\
\hline
\end{tabular}

Table 4. Convergence rates for the surrogate MI UN (unstructured) and MI FAO(4) (factor analytic) models as a function of balancedness of $n_{i}$, the number of clusters $(5,10,20,50)$ and the between-cluster variability.

\begin{tabular}{|c|c|c|c|c|c|c|c|c|c|c|}
\hline \multirow[b]{2}{*}{ Model } & \multirow{2}{*}{$\begin{array}{c}\text { Convergence } \\
\text { category }\end{array}$} & \multirow{2}{*}{$\begin{array}{c}\text { Between- } \\
\text { cluster } \\
\text { variability } \gamma\end{array}$} & \multicolumn{4}{|c|}{$\begin{array}{c}\text { Small imbalance } \\
\tilde{n}_{i} \sim N\left(20,2.5^{2}\right) \\
\text { Number of clusters }\end{array}$} & \multicolumn{4}{|c|}{$\begin{array}{l}\text { Large imbalance } \\
\tilde{n}_{i} \sim N\left(20,5^{2}\right) \\
\text { Number of clusters }\end{array}$} \\
\hline & & & 5 & 10 & 20 & 50 & 5 & 10 & 20 & 50 \\
\hline \multirow[t]{6}{*}{$\begin{array}{l}\text { Surrogate model, } \\
\text { MI UN }\end{array}$} & $\begin{array}{l}\text { Proper } \\
\quad \text { convergence }\end{array}$ & Small (0.1) & 0.164 & 0.941 & 1 & 1 & 0.189 & 0.946 & 0.999 & 0.999 \\
\hline & & Large (1) & 0.617 & 0.999 & 1 & 1 & 0.616 & 0.999 & 0.999 & 1 \\
\hline & $\begin{array}{l}\text { Convergence but } \\
\text { non-PD D/H } \\
\text { matrix }\end{array}$ & Small (0.1) & 0.811 & 0.059 & 0 & 0 & 0.770 & 0.054 & 0.001 & 0.001 \\
\hline & & Large (1) & 0.365 & 0.001 & 0 & 0 & 0.343 & 0.001 & 0.001 & 0 \\
\hline & Divergence & Small (0.1) & 0.025 & 0 & 0 & 0 & 0.042 & 0 & 0 & 0 \\
\hline & & Large (1) & 0.018 & 0 & 0 & 0 & 0.041 & 0 & 0 & 0 \\
\hline \multirow[t]{6}{*}{$\begin{array}{l}\text { Surrogate model, } \\
\text { MI FAO(4) }\end{array}$} & $\begin{array}{l}\text { Proper } \\
\quad \text { convergence }\end{array}$ & Small (0.1) & 0.813 & 0.998 & 1 & 1 & 0.817 & 0.996 & 0.999 & 0.999 \\
\hline & & Large (1) & 0.954 & 0.999 & 1 & 1 & 0.950 & 1 & 0.999 & 1 \\
\hline & $\begin{array}{l}\text { Convergence but } \\
\text { non-PD D/H } \\
\text { matrix }\end{array}$ & Small (0.1) & 0.077 & 0.002 & 0 & 0 & 0.072 & 0.003 & 0 & 0 \\
\hline & & Large (1) & 0.012 & 0.001 & 0 & 0 & 0.022 & 0 & 0.001 & 0 \\
\hline & Divergence & Small (0.1) & 0.109 & 0.002 & 0 & 0 & 0.111 & 0.001 & 0.001 & 0 \\
\hline & & Large (1) & 0.029 & 0.001 & 0 & 0 & 0.028 & 0 & 0 & 0 \\
\hline
\end{tabular}




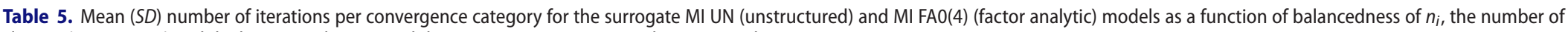
clusters $(5,10,20,50)$ and the between-cluster variability. Note. - : quantity cannot be computed.

\begin{tabular}{|c|c|c|c|c|c|c|c|c|c|c|}
\hline \multirow[b]{2}{*}{ Model } & \multirow[b]{2}{*}{$\begin{array}{l}\text { Convergence } \\
\text { category }\end{array}$} & \multirow{2}{*}{$\begin{array}{l}\text { Between- } \\
\text { cluster } \\
\text { variability } \gamma\end{array}$} & \multicolumn{4}{|c|}{$\begin{array}{c}\text { Small imbalance } \\
\tilde{n}_{i} \sim N(M=20, S D=2.5) \\
\text { Number of clusters }\end{array}$} & \multicolumn{4}{|c|}{$\begin{array}{c}\text { Large imbalanc } \\
\tilde{n}_{i} \sim N(M=20, S D=5) \\
\text { Number of clusters }\end{array}$} \\
\hline & & & 5 & 10 & 20 & 50 & 5 & 10 & 20 & 50 \\
\hline \multirow{6}{*}{$\begin{array}{l}\text { Surrogate model, } \\
\text { MI UN }\end{array}$} & Proper convergence & Small (0.1) & $1(0)$ & $1(0)$ & $1(0)$ & $1(0)$ & $1(0)$ & $1(0)$ & $1(0)$ & $1(0)$ \\
\hline & & Large (1) & $1(0)$ & $1(0)$ & $1(0)$ & $1(0)$ & $1(0)$ & $1(0)$ & $1(0)$ & $1(0)$ \\
\hline & $\begin{array}{l}\text { Convergence but } \\
\text { non-PD D/H matrix }\end{array}$ & Small (0.1) & $8.19(4.61)$ & $3.68(1.35)$ & - & - & $8.41(17.51)$ & $3.87(1.51)$ & $9.00(-)$ & $8.00(-)$ \\
\hline & & Large (1) & $6.20(3.79)$ & $5.00(0)$ & - & - & $6.15(4.48)$ & $5.00(-)$ & $2.50(2.12)$ & - \\
\hline & Divergence & Small (0.1) & 66.93(82.89) & - & - & - & $56.49(12.16)$ & - & - & - \\
\hline & & Large (1) & $55.26(8.29)$ & - & - & - & $54.96(17.06)$ & - & - & - \\
\hline \multirow{6}{*}{$\begin{array}{l}\text { Surrogate model, } \\
\text { MI FA0(4) }\end{array}$} & Proper convergence & Small (0.1) & $4.24(2.91)$ & $1.22(1.08)$ & $1(0)$ & $1(0)$ & $4.04(2.66)$ & $1.19(0.99)$ & $1(0)$ & $1(0)$ \\
\hline & & Large (1) & $2.06(1.91)$ & $1.00(0.07)$ & $1(0)$ & $1(0)$ & $2.04(1.90)$ & $1.00(0.06)$ & $1(0)$ & $1(0)$ \\
\hline & $\begin{array}{l}\text { Convergence but } \\
\text { non-PD D/H matrix }\end{array}$ & Small (0.1) & $5.92(1.94)$ & $4.50(1.38)$ & - & - & $5.87(1.99)$ & $4.89(1.17)$ & - & - \\
\hline & & Large (1) & $4.92(1.19)$ & $4.00(-)$ & - & - & $4.55(1.30)$ & - & $13.50(10.61)$ & - \\
\hline & Divergence & Small (0.1) & $21.25(12.83)$ & $33.60(7.27)$ & - & - & 22.18(13.28) & $38.75(10.05)$ & $19.00(-)$ & $56.00(-)$ \\
\hline & & Large (1) & $35.01(12.00)$ & $37.00(0)$ & - & - & $32.43(12.87)$ & - & - & - \\
\hline
\end{tabular}




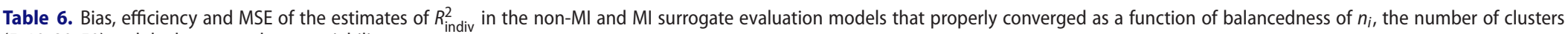
$(5,10,20,50)$ and the between-cluster variability.

\begin{tabular}{|c|c|c|c|c|c|c|c|c|c|c|c|c|c|c|}
\hline \multirow[b]{2}{*}{ Model } & \multirow[b]{2}{*}{ Measure } & \multirow{2}{*}{$\begin{array}{c}\text { Between- } \\
\text { cluster } \\
\text { variability }(\gamma)\end{array}$} & \multicolumn{4}{|c|}{$\begin{array}{l}\text { Balanced (equal } n_{i} \text { ) } \\
\text { Number of clusters }\end{array}$} & \multicolumn{4}{|c|}{$\begin{array}{c}\text { Small imbalance } \\
\tilde{n}_{i} \sim N\left(20,2.5^{2}\right) \\
\text { Number of clusters }\end{array}$} & \multicolumn{4}{|c|}{$\begin{array}{c}\text { Large imbalance } \\
\tilde{n}_{i} \sim N\left(20,5^{2}\right) \\
\text { Number of clusters }\end{array}$} \\
\hline & & & 5 & 10 & 20 & 50 & 5 & 10 & 20 & 50 & 5 & 10 & 20 & 50 \\
\hline \multirow{6}{*}{$\begin{array}{l}\text { Surrogate model, } \\
\text { non-MI, UN }\end{array}$} & Bias & Small (0.1) & -0.001 & -0.001 & -0.002 & 0.001 & -0.014 & 0.001 & -0.001 & 0.001 & 0.009 & 0.001 & -0.002 & 0.001 \\
\hline & & Large (1) & -0.005 & -0.001 & -0.001 & -0.001 & -0.009 & -0.001 & 0.001 & -0.001 & -0.009 & -0.001 & 0.001 & -0.001 \\
\hline & Efficiency & Small (0.1) & 0.080 & 0.052 & 0.037 & 0.023 & 0.072 & 0.053 & 0.037 & 0.023 & 0.067 & 0.052 & 0.038 & 0.023 \\
\hline & & Large (1) & 0.072 & 0.053 & 0.037 & 0.024 & 0.071 & 0.052 & 0.037 & 0.024 & 0.073 & 0.052 & 0.037 & 0.024 \\
\hline & MSE & Small (0.1) & 0.006 & 0.003 & 0.001 & 0.001 & 0.005 & 0.003 & 0.001 & 0.001 & 0.004 & 0.003 & 0.001 & 0.001 \\
\hline & & Large (1) & 0.005 & 0.003 & 0.001 & 0.001 & 0.005 & 0.003 & 0.001 & 0.001 & 0.005 & 0.003 & 0.001 & 0.001 \\
\hline \multirow{6}{*}{$\begin{array}{r}\text { Surrogate model, } \\
\text { non-MI, FAO(4) }\end{array}$} & Bias & Small (0.1) & -0.001 & -0.009 & -0.002 & 0.001 & -0.001 & 0.001 & -0.001 & 0.001 & -0.001 & 0.001 & -0.002 & 0.001 \\
\hline & & Large (1) & -0.006 & -0.001 & -0.001 & -0.001 & -0.007 & -0.001 & 0.001 & -0.001 & -0.007 & -0.001 & 0.001 & -0.001 \\
\hline & Efficiency & Small (0.1) & 0.074 & 0.052 & 0.037 & 0.023 & 0.076 & 0.053 & 0.037 & 0.023 & 0.072 & 0.051 & 0.038 & 0.023 \\
\hline & & Large (1) & 0.073 & 0.053 & 0.037 & 0.024 & 0.072 & 0.053 & 0.037 & 0.026 & 0.074 & 0.052 & 0.038 & 0.027 \\
\hline & MSE & Small (0.1) & 0.006 & 0.003 & 0.001 & 0.001 & 0.006 & 0.003 & 0.001 & 0.001 & 0.005 & 0.003 & 0.001 & 0.001 \\
\hline & & Large (1) & 0.005 & 0.003 & 0.001 & 0.001 & 0.005 & 0.003 & 0.001 & 0.001 & 0.006 & 0.003 & 0.001 & 0.001 \\
\hline \multirow{6}{*}{$\begin{array}{l}\text { Surrogate model, } \\
\text { MI UN }\end{array}$} & Bias & Small (0.1) & - & - & - & - & -0.005 & 0.001 & -0.001 & 0.003 & 0.008 & 0.004 & -0.001 & 0.004 \\
\hline & & Large (1) & - & - & - & - & -0.005 & 0.002 & 0.002 & 0.001 & -0.005 & 0.001 & 0.004 & 0.001 \\
\hline & Efficiency & Small (0.1) & - & - & - & - & 0.089 & 0.066 & 0.047 & 0.030 & 0.095 & 0.074 & 0.057 & 0.040 \\
\hline & & Large (1) & - & - & - & - & 0.087 & 0.065 & 0.047 & 0.031 & 0.098 & 0.076 & 0.057 & 0.043 \\
\hline & MSE & Small (0.1) & - & - & - & - & 0.008 & 0.004 & 0.002 & 0.001 & 0.009 & 0.005 & 0.003 & 0.002 \\
\hline & & Large (1) & - & - & - & - & 0.008 & 0.004 & 0.002 & 0.001 & 0.010 & 0.006 & 0.003 & 0.002 \\
\hline \multirow{6}{*}{$\begin{array}{l}\text { Surrogate model, } \\
\text { MI FAO(4) }\end{array}$} & Bias & Small (0.1) & - & - & - & - & -0.001 & 0.001 & -0.001 & 0.003 & 0.003 & 0.003 & -0.001 & 0.004 \\
\hline & & Large (1) & - & - & - & - & -0.005 & 0.002 & 0.002 & 0.001 & -0.005 & 0.001 & 0.004 & 0.001 \\
\hline & Efficiency & Small (0.1) & - & - & - & - & 0.090 & 0.066 & 0.047 & 0.030 & 0.097 & 0.074 & 0.057 & 0.040 \\
\hline & & Large (1) & - & - & - & - & 0.097 & 0.065 & 0.047 & 0.031 & 0.099 & 0.076 & 0.057 & 0.043 \\
\hline & MSE & Small (0.1) & - & - & - & - & 0.008 & 0.004 & 0.002 & 0.001 & 0.009 & 0.006 & 0.003 & 0.002 \\
\hline & & Large (1) & - & - & - & - & 0.008 & 0.004 & 0.002 & 0.001 & 0.010 & 0.006 & 0.003 & 0.002 \\
\hline
\end{tabular}

Note: UN = unstructured; FA0(4) = factor analytic. 


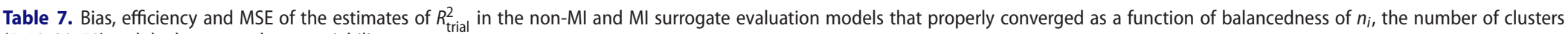
$(5,10,20,50)$ and the between-cluster variability.

\begin{tabular}{|c|c|c|c|c|c|c|c|c|c|c|c|c|c|c|}
\hline \multirow[b]{2}{*}{ Model } & \multirow[b]{2}{*}{ Measure } & \multirow{2}{*}{$\begin{array}{c}\text { Between- } \\
\text { cluster } \\
\text { variability }(\gamma)\end{array}$} & \multicolumn{4}{|c|}{$\begin{array}{l}\text { Balanced (equal } n_{i} \text { ) } \\
\text { Number of clusters }\end{array}$} & \multicolumn{4}{|c|}{$\begin{array}{c}\text { Small imbalance } \\
\tilde{n}_{i} \sim N\left(20,2.5^{2}\right) \\
\text { Number of clusters }\end{array}$} & \multicolumn{4}{|c|}{$\begin{array}{c}\text { Large imbalance } \\
\tilde{n}_{i} \sim N\left(20,5^{2}\right) \\
\text { Number of clusters }\end{array}$} \\
\hline & & & 5 & 10 & 20 & 50 & 5 & 10 & 20 & 50 & 5 & 10 & 20 & 50 \\
\hline \multirow{6}{*}{$\begin{array}{l}\text { Surrogate model, } \\
\text { non-MI, UN }\end{array}$} & Bias & Small (0.1) & -0.152 & -0.097 & -0.041 & -0.019 & -0.168 & -0.093 & -0.036 & -0.016 & -0.174 & -0.094 & -0.034 & -0.002 \\
\hline & & Large (1) & -0.168 & -0.075 & -0.031 & -0.013 & -0.170 & -0.074 & -0.031 & -0.012 & -0.152 & -0.074 & -0.029 & -0.009 \\
\hline & Efficiency & Small (0.1) & 0.254 & 0.224 & 0.182 & 0.111 & 0.246 & 0.229 & 0.184 & 0.112 & 0.280 & 0.232 & 0.187 & 0.114 \\
\hline & & Large (1) & 0.248 & 0.219 & 0.158 & 0.095 & 0.239 & 0.222 & 0.157 & 0.096 & 0.248 & 0.221 & 0.157 & 0.095 \\
\hline & MSE & Small (0.1) & 0.087 & 0.059 & 0.035 & 0.013 & 0.088 & 0.061 & 0.035 & 0.013 & 0.108 & 0.063 & 0.036 & 0.013 \\
\hline & & Large (1) & 0.089 & 0.053 & 0.026 & 0.009 & 0.086 & 0.055 & 0.026 & 0.009 & 0.084 & 0.054 & 0.025 & 0.009 \\
\hline \multirow{6}{*}{$\begin{array}{r}\text { Surrogate model, } \\
\text { non-MI, FAO(4) }\end{array}$} & Bias & Small (0.1) & -0.139 & -0.090 & -0.071 & -0.035 & -0.157 & -0.088 & -0.061 & -0.032 & -0.151 & -0.082 & -0.057 & -0.018 \\
\hline & & Large (1) & -0.099 & -0.077 & -0.060 & -0.024 & -0.109 & -0.073 & -0.063 & -0.024 & -0.076 & -0.071 & -0.058 & -0.020 \\
\hline & Efficiency & Small (0.1) & 0.267 & 0.233 & 0.203 & 0.121 & 0.269 & 0.238 & 0.197 & 0.122 & 0.273 & 0.237 & 0.201 & 0.125 \\
\hline & & Large (1) & 0.272 & 0.232 & 0.178 & 0.101 & 0.269 & 0.236 & 0.179 & 0.100 & 0.266 & 0.234 & 0.177 & 0.101 \\
\hline & MSE & Small (0.1) & 0.090 & 0.062 & 0.046 & 0.016 & 0.096 & 0.064 & 0.043 & 0.016 & 0.097 & 0.063 & 0.044 & 0.016 \\
\hline & & Large (1) & 0.084 & 0.060 & 0.035 & 0.011 & 0.084 & 0.061 & 0.036 & 0.011 & 0.076 & 0.060 & 0.035 & 0.011 \\
\hline \multirow{6}{*}{$\begin{array}{l}\text { Surrogate model, } \\
\text { MI UN }\end{array}$} & Bias & Small (0.1) & - & - & - & - & -0.186 & -0.080 & -0.034 & -0.016 & -0.193 & -0.087 & -0.036 & -0.022 \\
\hline & & Large (1) & - & - & - & - & -0.165 & -0.071 & -0.031 & -0.012 & -0.163 & -0.072 & -0.032 & -0.018 \\
\hline & Efficiency & Small (0.1) & - & - & - & - & 0.255 & 0.226 & 0.172 & 0.105 & 0.257 & 0.229 & 0.174 & 0.128 \\
\hline & & Large (1) & - & - & - & - & 0.244 & 0.221 & 0.155 & 0.095 & 0.254 & 0.222 & 0.160 & 0.108 \\
\hline & MSE & Small (0.1) & - & - & - & - & 0.099 & 0.057 & 0.031 & 0.011 & 0.103 & 0.060 & 0.031 & 0.017 \\
\hline & & Large (1) & - & - & - & - & 0.087 & 0.054 & 0.025 & 0.009 & 0.091 & 0.054 & 0.027 & 0.012 \\
\hline \multirow{6}{*}{$\begin{array}{l}\text { Surrogate model, } \\
\text { MI FAO(4) }\end{array}$} & Bias & Small (0.1) & - & - & - & - & -0.258 & -0.088 & -0.034 & -0.016 & -0.266 & -0.094 & -0.036 & -0.022 \\
\hline & & Large (1) & - & - & - & - & -0.193 & -0.071 & -0.031 & -0.012 & -0.193 & -0.072 & -0.032 & -0.018 \\
\hline & Efficiency & Small (0.1) & - & - & - & - & 0.251 & 0.228 & 0.172 & 0.105 & 0.251 & 0.233 & 0.174 & 0.128 \\
\hline & & Large (1) & - & - & - & - & 0.253 & 0.221 & 0.155 & 0.095 & 0.261 & 0.222 & 0.160 & 0.108 \\
\hline & MSE & Small (0.1) & - & - & - & - & 0.130 & 0.060 & 0.031 & 0.011 & 0.134 & 0.063 & 0.031 & 0.017 \\
\hline & & Large (1) & - & - & - & - & 0.101 & 0.054 & 0.025 & 0.009 & 0.106 & 0.054 & 0.027 & 0.011 \\
\hline
\end{tabular}

Note: UN = unstructured; FA0(4) = factor analytic. 
For example, in cluster 1 there were five observations for $S$ and $T$ in treatment group $Z=-1$ and 11 observations in treatment group $Z=1$. Thus, the data of 13 and 7 patients are imputed in cluster 1 for $Z=-1$ and $Z=1$, respectively.

Proper multivariate imputations were conducted using the default Markov chain Monte Carlo method [11] in SAS with a non-informative prior (Jeffreys). The imputation model included $S, T$, and $Z$. The imputation model was run 'by cluster'. This is a valid approach here, provided that the number of clusters increases sufficiently slowly relative to the number of subjects per cluster. A total of 200 burn-in iterations were used (i.e. the number of initial iterations before the first iteration for a chain), and the number of iterations between the imputations in a chain equalled 100. A total of 3 imputations were conducted for each dataset. Thus in total, 24,000 data sets were considered in the analyses (i.e. 4 (number of $N$ ) $\cdot 2$ (number of $\gamma$ ) $\cdot 1000$ (number of runs) 3 (number of imputations)) for both the small imbalance and the large imbalance scenarios.

The 'balanced' data were subsequently analysed by fitting Model (1) using the UN and FA0(4) parametrizations for the $\mathbf{D}$ matrix (Section 2.2.3). The outcomes of interest were model convergence and the number of required iterations to achieve convergence (Section 2.1). In addition, the bias, efficiency (standard deviation of the estimate) and mean-squared errors of the estimates of the $R_{\text {trial }}^{2}$ and $R_{\text {indiv }}^{2}$ metrics were evaluated for the surrogate evaluation models with MI using an unstructured (UN MI) and non-diagonal factor analytic structure with 4 factors (MI FA0(4)) for the D matrix. The focus was on $R_{\text {trial }}^{2}$ and $R_{\text {indiv }}^{2}$ rather than on the fixed-effects and variance components because the coefficients of determination are the main quantities of interest in a surrogate evaluation context.

\subsection{Results}

Table 4 shows the convergence rates for the MI UN and MI FA0(4) models. Compared to what was the case for the surrogate evaluation models in the unbalanced non-MI scenarios, the rates of proper and overall convergence were substantially higher in both the MI UN and MI FA0(4) scenarios - and this was particularly so when $N$ was small. The use of MI to make the unbalanced data balanced was thus a successful strategy to improve convergence and reduce divergence. In line with the results discussed in Section 2.3, proper convergence was always achieved after 1 iteration in the MI UN scenario but not in the MI FA0(4) scenario (see Table 5). Nonetheless, the number of required iterations to achieve convergence was substantially reduced in the unbalanced MI FA0(4) scenarios compared to what was the case in the non-MI unbalanced scenarios (compare Tables 2 and 5).

Tables 6 and 7 show the bias, efficiency, and MSE of the estimates of $R_{\text {indiv }}^{2}$ and $R_{\text {trial }}^{2}$, respectively, in the non-MI and MI settings that properly converged. As expected, the bias, efficiency and MSE in the estimation of both $R_{\text {indiv }}^{2}$ and $R_{\text {trial }}^{2}$ improved when the number of clusters increased. With respect to the estimation of $R_{\text {indiv }}^{2}$, the bias was low in all scenarios but the efficiency and MSE were poorer in the MI scenarios compared to the non-MI scenarios. In contrast to $R_{\text {indiv }}^{2}$, the bias, efficiency and MSE were of similar magnitude in the MI and non-MI scenarios. Only when $N=5$ did the bias in the estimation of $R_{\text {trial }}^{2}$ tend to be substantially higher in the MI scenario compared to the non-MI scenario. Note that the bias was negative in all scenarios, indicating that the true $R_{\text {trial }}^{2}$ tends to be somewhat underestimated.

Furthermore, the bias and MSE in the estimation of $R_{\text {indiv }}^{2}$ was smaller compared to what was observed for $R_{\text {trial }}^{2}$, and the efficiency somewhat lower, because there is less replication than for the individual-level quantity.

\section{Case study}

The results in Section 3 indicated that the use of MI to balance an unbalanced data set (prior to fitting the mixed-effects model) reduces model convergence issues. In this section, this method is applied to a real-life data set, the age-related macular degeneration trial. 


\subsection{An age-related macular degeneration trial}

Age-related macular degeneration (ARMD) is a condition in which patients progressively lose vision.[12] In the ARMD trial, patients were randomly allocated to two treatment conditions: placebo and the experimental treatment (interferon- $\alpha$ ). Treatment efficacy was evaluated using changes in visual acuity over time. Visual acuity was measured as the total number of letters that were correctly read using standardized vision charts.

The ARMD trial was analysed earlier in a surrogate evaluation context.[4,13] The idea is to examine whether the change in visual acuity after 24 weeks is an appropriate surrogate for the change in visual acuity after 52 weeks. The ARMD data are included in the R library Surrogate, which can be downloaded at http://cran.r-project.org/web/packages/Surrogate/index.html.

\subsection{Sample descriptives}

The ARMD trial enrolled a total of 181 patients from 36 centres. Here, the unit of analysis (i.e. the clustering variable) is centre. Centres that enrolled less than 5 patients (19 centres in total) were discarded from the analyses to avoid problems during the MI phase (recall that the imputations are conducted for each centre separately).

The data of 119 patients from 17 centres were analysed. On average, there were 7 patients per centre. A total of $6,2,4,1,2$, and 1 centres had 5, 6, 4, 7, 8, 9, and 18 patients, respectively. In the centre with 18 patients, 9 patients received placebo and 9 patients received interferon- $\alpha$.

\subsection{Analysis}

The same procedure that was described in Section 3 to obtain balanced data sets (using MI) was employed here. Thus, in all centre by treatment groups that had less than 9 patients, data were imputed to achieve balance. The imputations were conducted for each of the centres separately, using $S$, $T$, and $Z$ in the imputation model.

A total of 1000 imputations were conducted. For each of the balanced data sets, Model (1) was fitted using $S=$ change in visual acuity after 24 weeks and $T=$ change in visual acuity after 52 weeks. Both the FA0(4) and UN covariance parametrizations for $\mathbf{D}$ were used.

The key outcome of interest was model convergence (using the same convergence categories as were defined in Section 2). In addition, the trial- and individual-level coefficients of surrogacy $\left(R_{\text {trial }}^{2}\right.$ and $R_{\text {indiv }}^{2}$ ) were computed for all data sets.

\subsection{Results}

\subsubsection{Convergence rates}

When Model (1) was fitted to the non-imputed data of the case study (both the entire ARMD data set and the data set that only included centres that enrolled at least five patients were considered), convergence issues occurred. In particular, the models that used the UN parametrization for the D matrix did not converge and the models that used the FA0(4) parametrization converged to a non-PD D/H matrix.

Table 8 shows the convergence rates that were obtained when the MI-based approaches were used. Overall convergence was high and equalled $100 \%$ and $96.9 \%$ in the MI UN and MI FA0(4) scenarios,

Table 8. Convergence rates for the ARMD data (using MI UN and $\mathrm{MI} \mathrm{FAO(4)} \mathrm{to} \mathrm{restore} \mathrm{'balance'} \mathrm{in} \mathrm{cluster} \mathrm{size).}$

\begin{tabular}{lcc}
\hline & MI UN & MI FA0(4) \\
\hline Proper convergence & 0.701 & 0.944 \\
Convergence but non-PD D/H matrix & 0.299 & 0.015 \\
Divergence & 0 & 0.041 \\
\hline
\end{tabular}

Note: UN = unstructured; FAO(4) = factor analytic. 

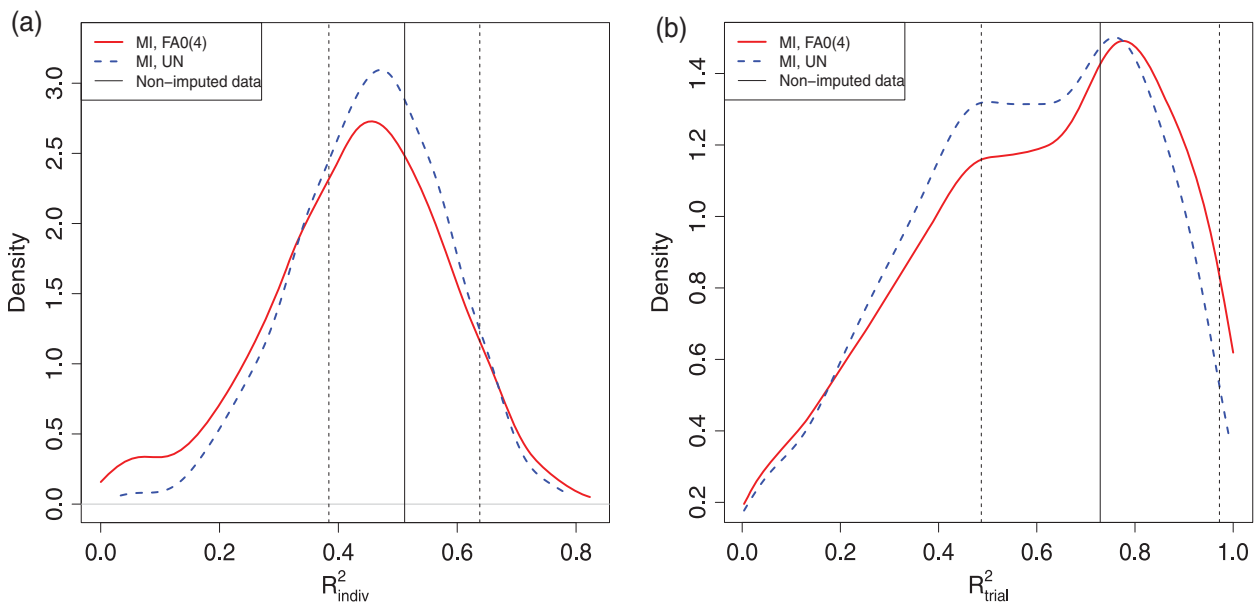

Figure 1. Densities of $\hat{R}_{\text {indiv }}^{2}$ (left) and $\hat{R}_{\text {trial }}^{2}$ (right) in the ARMD data sets using the MI UN and MI FAO(4) strategies. The vertical full lines are the point estimates for $\hat{R}_{\text {indiv }}^{2}$ and $\hat{R}_{\text {trial }}^{2}$ in the non-imputed data set (using a two-stage modelling approach) and the vertical dashed lines are the $95 \%$ confidence intervals.

respectively. Furthermore, the use of the MI FA0(4) strategy led to higher rates of proper convergence compared to the MI UN strategy ( $94.4 \%$ versus $70.1 \%$, respectively), whereas the MI UN strategy led to lower divergence rates compared to the MI FA0(4) scenario ( $0 \%$ versus $4.1 \%$, respectively). These results are fully in line with the results that were obtained in the simulation studies detailed above.

\subsubsection{Coefficients of surrogacy}

The densities of the trial- and individual-level surrogacy estimates $\left(\hat{R}_{\text {trial }}^{2}\right.$ and $\hat{R}_{\text {indiv }}^{2}$, respectively) using the MI UN and MI FA0(4) strategies are shown in Figure 1.

The mean $\hat{R}_{\text {trial }}^{2}$ and $\hat{R}_{\text {indiv }}^{2}$ equalled 0.573 and 0.453 for the MI UN models, and 0.597 and 0.431 for the MI FA0(4) models, respectively. To establish a frame of reference against which these estimates can be compared, the two-stage equivalent of Model (1) was fitted to the non-imputed ARMD data (a simplified approach was used because Model (1) did not converge). In particular, in the first stage the following bivariate model is fitted:

$$
\begin{aligned}
& S_{i j}=\mu_{S}+\alpha_{i} Z_{i j}+\varepsilon_{S i j}, \\
& T_{i j}=\mu_{T}+\beta_{i} Z_{i j}+\varepsilon_{T i j},
\end{aligned}
$$

where $\mu_{S}, \mu_{T}$ are the common intercepts for $S$ and $T, \alpha_{i}, \beta_{i}$ are the fixed trial-specific treatment effects for $S$ and $T$, and the other parameters are the same as defined above. In the second stage, $\hat{\beta}_{i}$ is regressed on $\hat{\alpha}_{i}$. The classical coefficient of determination of the fitted stage 2 model provides an estimate of $R_{\text {trial }}^{2}$ and $R_{\text {indiv }}^{2}=\operatorname{corr}\left(\varepsilon_{S i j}, \varepsilon_{T i j}\right)^{2}$ (for details on simplifying model-fitting strategies, see [14]). This analysis yielded $\hat{R}_{\text {trial }}^{2}=0.729$ with $C I_{95 \%}=[0.487 ; 0.972]$ and $\hat{R}_{\text {indiv }}^{2}=0.512$ with $C I_{95 \%}=[0.384 ; 0.639]$. The vertical solid and dashed lines in Figure 1 indicate these point estimates and their $95 \%$ confidence intervals, respectively.

Overall, the results indicate that there was an acceptable agreement between the trial- and individual-level surrogacy estimates that were obtained in the MI-based and non-MI based approaches, though it should be noted that the variability of the MI-based $\hat{R}_{\text {trial }}^{2}$ and $\hat{R}_{\text {indiv }}^{2}$ values was large (see also Figure 1). For example, the 2.5th and 97.5th percentile values of $\hat{R}_{\text {trial }}^{2}$ equalled $P c_{2.5}=$ $0.078, P c_{97.5}=0.941$ and $P c_{2.5}=0.069, P c_{97.5}=0.985$ in the MI UN and MI FA0(4) scenarios, respectively. The large variability of these estimates should be evaluated in light of the relatively small number of clusters and patients in the ARMD data set. In addition, there were large imbalances in the cluster sizes in the ARMD data set. For example, 7 out of the 17 centres that were available for 
analysis had only 5 patients and thus the ratio of the available data relative to the data that had to be imputed in these centres was small ( 5 versus 13 patients). It seems reasonable to assume that the variability of $\hat{R}_{\text {indiv }}^{2}$ and $\hat{R}_{\text {trial }}^{2}$ will be smaller when this ratio is higher, though additional analyses are needed to substantiate this claim. Furthermore, the results showed that the use of the MI UN and MI FA0(4) strategies yielded nearly identical estimates for both coefficients of surrogacy.

\section{Discussion}

In line with earlier research,[3,7] the convergence rates of the mixed-effects models were found to be substantially higher when the number of available clusters increased and when the size of the between-cluster variability $\mathbf{D}$ was large relative to the residual variability $\boldsymbol{\Sigma}$. The present simulation study further extend these findings by showing that an imbalance in cluster size was associated with more model convergence issues. This was particularly the case when the model at hand had a complex hierarchical structure. The divergence rates were higher when the imbalance in cluster size was larger, and the use of MI to make the unbalanced data sets balanced reduced model convergence issues. Bias in the estimation of $R_{\text {indiv }}^{2}$ was similar in the non-MI and MI scenarios, but the use of MI led to a decreased efficiency and increased MSE. With respect to the estimation of $R_{\text {trial }}^{2}$, bias, efficiency, and MSE were comparable in all scenarios where there were more than five clusters available.

The combination of good convergence properties and satisfactory statistical properties of the estimators, as follows from our simulation study, also suggests that there is little risk of convergence to local optima. In addition, our experience from past data analyses and simulations (see, e.g. [4]) is in line with this finding.

With due caution, in scenarios where the convergence properties of the maximum likelihood estimator are poor (e.g. when $N$ and $\gamma$ are small), a forthright recommendation is to use MI with the Cholesky decomposition formulation of the variance-covariance matrix. Of course, there are secondary issues pertaining to for example, the target of inference, which may or may not place emphasis on the variance components. For example, in the present simulations, three model convergence categories were distinguished, that is (i) proper convergence, (ii) convergence but a non-PD D or $\mathbf{H}$ matrix, and (iii) divergence. The relevance of distinguishing between categories (i)-(ii) depends on the substantive research question at hand. In a surrogate validation context, the distinction is important because one is mainly interested in the variance components (e.g. $\mathbf{D}$ should be PD to guarantee that $\hat{R}_{\text {trial }}^{2}$ is within the unit interval). If one is merely interested in the fixed-effects (the marginal model), this distinction is unimportant because the marginal model can be used to make valid inferences regarding the fixed-effect parameters as long as the overall $\mathbf{V}$ matrix is PD.[2,10] Thus, in practice, a researcher who is mainly interested in making inferences regarding the random effects may opt for the strategy that leads to the highest rates of proper convergence (e.g. MI with FA0(4) for the $\mathbf{D}$ matrix), whereas a researcher who is mainly interested in the marginal model may opt for the strategy that leads to the highest rates of overall convergence (e.g. MI with UN for the D matrix).

Several alternative imputation models are potentially of use here - though any feasible model needs to be compatible with the analysis model. For example, hierarchical versions could be considered that take into account all three levels.[15] In our specific context, where typically there is a relatively small number of trials, with a good amount of replication per trial, also a trial-specific strategy is viable. The method we have proposed is both computationally convenient and has good convergence and statistical properties.

Some comments and suggestions for future research are in place. First, the convergence rates were relatively high for all models in all scenarios. For example, the proper convergence rates were close to $100 \%$ when the number of clusters exceeded 20 for all models in all scenarios (Tables 1 and 4). Obviously, the convergence rates that are obtained in a simulation study depend on the choice of the parameters that are used to generate the data. For example, in the present simulations the variance components in the $\mathbf{D}$ matrix that were used to generate the data were all relatively large. This choice was made to avoid model convergence problems that arise by hitting the boundary of the parameter 
space. When lower valued D matrices were used (keeping all other parameters constant), the convergence rates substantially decreased but the global pattern of the results in terms of the impact of an imbalance in cluster size on model divergence rates and the effect of the use of MI remained the same. This is further illustrated in Table A1 in the appendix (which shows the convergence rates for a scenario where $\gamma=0.01$, keeping all other parameters that were used to generate the data identical to the ones provided in Section 2) and in the supplementary materials.

In the supplementary materials, lower valued $\mathbf{D}$ matrices were used and a number of additional scenarios to introduce imbalance in cluster size and/or treatment allocation were considered. Furthermore, the (mean) cluster size that was used in the analyses in the supplementary materials was substantially lower and closer to the mean cluster size in the ARMD data set (i.e. mean $n=10$ instead of $n=20$ ). The results that were obtained in these additional scenarios were similar to the main results discussed above.

Note also that the focus of the current study was on linear mixed-effects models only. Further simulation studies would be needed to examine this issue in non-linear mixed-effects models.

Second, missing data frequently arise in a surrogate evaluation setting (i.e. the measurement of $T$ is by definition cumbersome, otherwise there would be no need for a surrogate) and in many other research settings. An advantage of the MI-based strategy proposed above is that it provides a natural framework to deal with unbalanced cluster sizes and missingness at the same time. This can be done in a flexible way, for example, it is straightforward to include covariates, such as the age of the patient or a post-randomization non-compliance measure in the imputation model whilst at the same time keeping the standard substantive Model (1).[9]

Finally, MI was used to augment the data and Newton-Raphson was used to conduct the optimization of the log-likelihood functions, but other choices are viable as well. For example, future studies may consider the use of EM to augment the data and/or Fisher scoring for the optimization. Furthermore, all simulation results discussed in the present paper were obtained using SAS, and it would be useful to evaluate whether similar results are obtained when other software tools (e.g. R, Stata, and MLwiN) are used.

\section{Disclosure statement}

No potential conflict of interest was reported by the authors.

\section{Funding}

Financial support from the IAP research network \#P7/06 of the Belgian Government (Belgian Science Policy) is gratefully acknowledged. Wim Van der Elst acknowledges funding from the European Seventh Framework programme FP7 2007 - 2013 under grant agreement Nr. 602552. Geert Molenberghs acknowledges funding from Intel, Janssen Pharmaceutica and by the Institute for the Promotion of Innovation through Science and Technology in Flanders (IWT).

\section{Supplemental data}

Supplemental data for this article can be accessed at doi:10.1080/00949655.2015.1103738.

\section{References}

[1] Lindstrom MJ, Bates DM. Newton-Raphson and EM algorithms for linear mixed-effects models for repeatedmeasures data. J Amer Statist Assoc. 1988;83:1014-1022.

[2] Verbeke G, Molenberghs G. Linear mixed models for longitudinal data. New York: Springer; 2000.

[3] Buyse M, Molenberghs G, Burzykowski T, Renard D, Geys H. The validation of surrogate endpoints in metaanalyses of randomized experiments. Biostatistics. 2000;1:49-67.

[4] Burzykowski T, Molenberghs G, Buyse M. The evaluation of surrogate endpoints. New York: Springer; 2005.

[5] Prentice RL. Surrogate endpoints in clinical trials: definition and operational criteria. Stat Med. 1989;8:431-440.

[6] Cortiñas AJ, Molenberghs G, Burzykowski T, Shkedy Z, Renard D. Choice of units of analysis and modeling strategies in multilevel hierarchical models. Comput Stat Data Anal. 2004;47:537-563. 
[7] Renard D, Geys H, Molenberghs G, Burzykowski T, Buyse M. Validation of surrogate endpoints in multiple randomized clinical trials with discrete outcomes. Biom J. 2002;44:921-935.

[8] Dempster AP, Laird NM, Rubin DB. Maximum likelihood from incomplete data via the EM algorithm (with discussion). J R Statist Soc Ser B. 1977;39:1-38.

[9] Molenberghs G, Kenward MG. Missing data in clinical studies. New York: Wiley; 2007.

[10] West BT, Welch KB, Galecki AT. Linear mixed models: a practical guide using statistical software. New York: Chapman \& Hall/CRC; 2007.

[11] Schafer JL. Analysis of incomplete multivariate data. New York: Chapman \& Hall; 1997.

[12] Pharmacological therapy for macular degeneration study group. Interferon $\alpha$-IIA is ineffective for patients with choroidal neovascularization secondary to age-related macular degeneration. Results of a prospective randomized placebo-controlled clinical trial. Arch Ophthalmol. 1997;115:865-872.

[13] Alonso A, VanderElst W, Molenberghs G, Buyse M, Burzykowski T. On the relationship between the causalinference and meta-analytic paradigms for the validation of surrogate endpoints. Biometrics. 2015;71:15-24.

[14] Tibaldi FS, Cortiñas AJ, Molenberghs G, Renard D, Burzykowski T, Buyse M, Parmar M, Stijnen T, Wolfinger R. Simplified hierarchical linear models for the evaluation of surrogate endpoints. J Statist Comput Simul. 2003;73:643-658.

[15] Carpenter J, Kenward MG. Multiple imputation and its application. New York: Wiley; 2014.

\section{Appendix}

Table A1. Convergence rates (using $\gamma=0.01$ ) for the random-intercept models, reduced surrogate models, and surrogate evaluation models as a function of balancedness of $n_{i}$ and the number of clusters $(5,10,20,50)$.

\begin{tabular}{|c|c|c|c|c|c|c|c|c|c|c|c|c|c|}
\hline \multirow[b]{2}{*}{ Model } & \multirow{2}{*}{$\begin{array}{c}\text { Convergence } \\
\text { category }\end{array}$} & \multicolumn{4}{|c|}{$\begin{array}{l}\text { Balanced (equal } n_{i} \text { ) } \\
\text { Number of clusters }\end{array}$} & \multicolumn{4}{|c|}{$\begin{array}{c}\text { Small imbalance } \\
\tilde{n}_{i} \sim N\left(20,2.5^{2}\right) \\
\text { Number of clusters }\end{array}$} & \multicolumn{4}{|c|}{$\begin{array}{c}\text { Large imbalance } \\
\tilde{n}_{i} \sim N\left(20,5^{2}\right) \\
\text { Number of clusters }\end{array}$} \\
\hline & & 5 & 10 & 20 & 50 & 5 & 10 & 20 & 50 & 5 & 10 & 20 & 50 \\
\hline Random & $\begin{array}{l}\text { Proper } \\
\quad \text { convergence }\end{array}$ & 0.656 & 0.797 & 0.910 & 0.982 & 0.656 & 0.812 & 0.910 & 0.982 & 0.651 & 0.798 & 0.903 & 0.981 \\
\hline \multirow[t]{2}{*}{ intercept } & $\begin{array}{c}\text { Convergence but } \\
\text { non-PD D/H }\end{array}$ & 0.344 & 0.203 & 0.081 & 0.018 & 0.344 & 0.188 & 0.090 & 0.018 & 0.349 & 0.202 & 0.097 & 0.019 \\
\hline & Divergence & 0 & 0 & 0 & 0 & 0 & 0 & 0 & 0 & 0 & 0 & 0 & 0 \\
\hline \multirow[t]{3}{*}{$\begin{array}{l}\text { Reduced } \\
\text { surrogate }\end{array}$} & $\begin{array}{l}\text { Proper } \\
\quad \text { convergence }\end{array}$ & 0.280 & 0.512 & 0.735 & 0.946 & 0.281 & 0.511 & 0.747 & 0.944 & 0.242 & 0.484 & 0.710 & 0.945 \\
\hline & $\begin{array}{c}\text { Convergence but } \\
\text { non-PD D/H }\end{array}$ & 0.720 & 0.488 & 0.265 & 0.054 & 0.654 & 0.487 & 0.253 & 0.056 & 0.614 & 0.503 & 0.289 & 0.055 \\
\hline & Divergence & 0 & 0 & 0 & 0 & 0 & 0 & 0 & 0 & 0.144 & 0.013 & 0.001 & 0 \\
\hline $\begin{array}{l}\text { Surrogate } \\
\text { model }\end{array}$ & $\begin{array}{l}\text { Proper } \\
\quad \text { convergence }\end{array}$ & 0 & 0.032 & 0.170 & 0.648 & 0.001 & 0.019 & 0.171 & 0.672 & 0.001 & 0.019 & 0.149 & 0.624 \\
\hline \multirow[t]{2}{*}{ non-MI, UN } & $\begin{array}{c}\text { Convergence but } \\
\text { non-PD D/H }\end{array}$ & 0.999 & 0.968 & 0.830 & 0.352 & 0.056 & 0.654 & 0.809 & 0.328 & 0.015 & 0.428 & 0.789 & 0.376 \\
\hline & Divergence & 0.010 & 0 & 0 & 0 & 0.943 & 0.327 & 0.020 & 0 & 0.984 & 0.553 & 0.062 & 0 \\
\hline $\begin{array}{l}\text { Surrogate } \\
\text { model }\end{array}$ & $\begin{array}{l}\text { Proper } \\
\quad \text { convergence }\end{array}$ & 0.281 & 0.457 & 0.674 & 0.918 & 0.280 & 0.454 & 0.634 & 0.918 & 0.307 & 0.467 & 0.629 & 0.898 \\
\hline \multirow[t]{2}{*}{ non-MI, FAO(4) } & $\begin{array}{c}\text { Convergence but } \\
\text { non-PD D/H }\end{array}$ & 0.064 & 0.063 & 0.047 & 0.023 & 0.052 & 0.047 & 0.050 & 0.024 & 0.054 & 0.044 & 0.059 & 0.028 \\
\hline & Divergence & 0.655 & 0.480 & 0.279 & 0.059 & 0.668 & 0.499 & 0.316 & 0.058 & 0.639 & 0.489 & 0.312 & 0.074 \\
\hline $\begin{array}{c}\text { Surrogate } \\
\text { model }\end{array}$ & $\begin{array}{l}\text { Proper } \\
\quad \text { convergence }\end{array}$ & - & - & - & - & 0.008 & 0.291 & 0.867 & 0.999 & 0.014 & 0.388 & 0.932 & 0.999 \\
\hline \multirow[t]{2}{*}{ MIUN } & $\begin{array}{c}\text { Convergence but } \\
\text { non-PD D/H }\end{array}$ & - & - & - & - & 0.963 & 0.709 & 0.133 & 0.001 & 0.942 & 0.612 & 0.068 & 0.001 \\
\hline & Divergence & - & - & - & - & 0.029 & 0 & 0 & 0 & 0.044 & 0 & 0 & 0 \\
\hline $\begin{array}{c}\text { Surrogate } \\
\text { model }\end{array}$ & $\begin{array}{l}\text { Proper } \\
\quad \text { convergence }\end{array}$ & - & - & - & - & 0.428 & 0.796 & 0.984 & 1 & 0.439 & 0.848 & 0.989 & 0.999 \\
\hline \multirow[t]{2}{*}{ MI FA0(4) } & $\begin{array}{c}\text { Convergence but } \\
\text { non-PD D/H }\end{array}$ & - & - & - & - & 0.061 & 0.051 & 0.009 & 0 & 0.067 & 0.044 & 0.008 & 0.001 \\
\hline & Divergence & - & - & - & - & 0.511 & 0.154 & 0.007 & 0 & 0.494 & 0.108 & 0 & 0 \\
\hline
\end{tabular}

Note. UN = unstructured; FA0(4) = factor analytic. 